\title{
Guidelines for periodontal care and follow-up during orthodontic treatment in adolescents and young adults
}

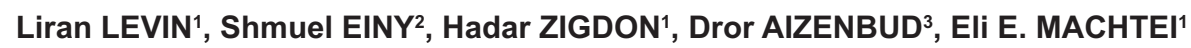 \\ 1- DMD, Department of Periodontology, School of Graduate Dentistry, Rambam Health Care Campus; Faculty of Medicine, Technion - Israel Institute of \\ Technology, Haifa, Israel. \\ 2- DMD, MSc, Orthodontic and Craniofacial Center, School of Graduate Dentistry, Rambam Health Care Campus, Haifa, Israel. \\ 3- DMD, MSc, Orthodontic and Craniofacial Center, School of Graduate Dentistry, Rambam Health Care Campus; Faculty of Medicine, Technion - Israel \\ Institute of Technology, Haifa, Israel.
}

Corresponding address: Dr. Liran Levin - Department of Periodontology, School of Graduate Dentistry - Rambam Health Care Campus, P.O. Box 9602 - Haifa 31096, Israel - Phone: 972-4-8542983 - e-mail: liranl@tx.technion.ac.il

Received: August 14, 2010 - Accepted: April 03, 2011

\section{ABSTRACT}

\begin{abstract}
Agressive periodontitis is characterized by non-contributory medical history, rapid attachment loss and bone destruction and familial aggregation of cases. Aggressive periodontitis (both localized and generalized) is usually diagnosed in a young population. This is frequently the age that an orthodontic care is provided to this population. The aim of the present paper is to draw guidelines for periodontal evaluation and monitoring prior to and during active orthodontic treatment. Strict adherence to these guidelines as a routine protocol for periodontal examination prior, during and following orthodontic treatment may dramatically decrease the severity and improve the prognosis of patients with aggressive periodontitis in orthodontic clinics.
\end{abstract}

Key words: Aggressive periodontitis. Diagnosis. Bone loss. Gingival recession. Gingivitis. Radiography.

\section{INTRODUCTION}

The term "aggressive periodontitis" was first introduced at the World Workshop on Periodontal Disease Classification ${ }^{3}$. Previously, different clinical patterns of significant loss of alveolar bone and attachment in young adults were known as early onset or juvenile periodontitis. Aggressive periodontitis is characterized by non-contributory medical history, rapid attachment loss and bone destruction and familial aggregation of cases. Localized aggressive periodontitis usually present circumpubertal onset and localized first molar/ incisor presentation with interproximal attachment loss on at least two permanent teeth, one of which is a first molar, and involving no more than two teeth other than first molars and incisors. While generalized aggressive periodontitis presents generalized interproximal attachment loss affecting at least three permanent teeth other than first molars and incisors with pronounced episodic nature of the destruction of attachment and alveolar bone ${ }^{3}$.

The prevalence of localized aggressive periodontitis varies greatly; from $0.1-0.2 \%$ reported in European populations ${ }^{6,13}$ to $5 \%$ in Hispanic populations ${ }^{1}$, up to $10 \%$ for African-Americans in the US. Löe and Brown ${ }^{9}$ (1991) estimated that African-American teenagers are 15 times more likely to present aggressive periodontitis than Caucasians. However, a large variation still exists in different African populations. Harley and Floyd ${ }^{5}$ (1988) report a prevalence of $0.8 \%$ among Nigerian teenagers, and Arowojolu and Nwokorie ${ }^{4}$ (1997) found a prevalence of $1.6 \%$ among $17-34$ years olds in a Nigerian hospital clinic. More recently, Albandar, et al. ${ }^{2}$ (2002) report among 12-25-yearold who attended Ugandan schools $6.5 \%$ aggressive periodontitis (either localized or generalized) and $22 \%$ incidental aggressive lesions. Similarly, other ethnical groups show high prevalence of this disease; in a recent study from Israel, aggressive periodontitis was found in $5.9 \%$ of the subjects ${ }^{7}$. 
Aggressive periodontitis (both localized and generalized) is usually diagnosed in a young population. This is frequently the age that an orthodontic care is provided to this population.

The first molar, usually involved in aggressive periodontitis, is also the tooth frequently in use as an abutment for different active or passive orthodontic appliances. Several orthodontic distalization appliances used in Class II malocclusions, such as headgear, pendulum or distal jet, incorporated
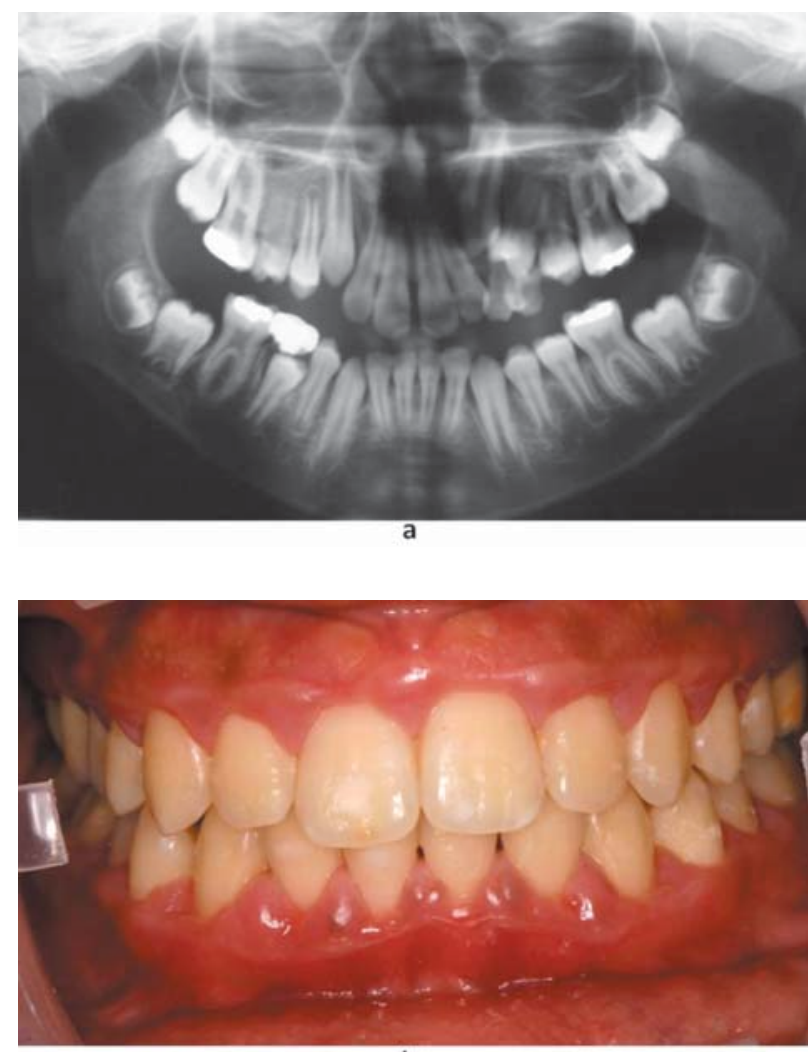

bilateral orthodontic molars bands on upper first molars. Lip bumper, which is a lower distalization appliance, is also anchored bilaterally to lower molar bands. Maxillary expanders, such as Haas or Hyrax appliances, are soldered to maxillary molars bands. Uni/bilateral space maintainers, frequently used in pediatric dentistry and orthodontics, include maxillary or mandibular molar bands soldered to a thick orthodontic wire as can be presented by Nance appliance, transpalatal arch
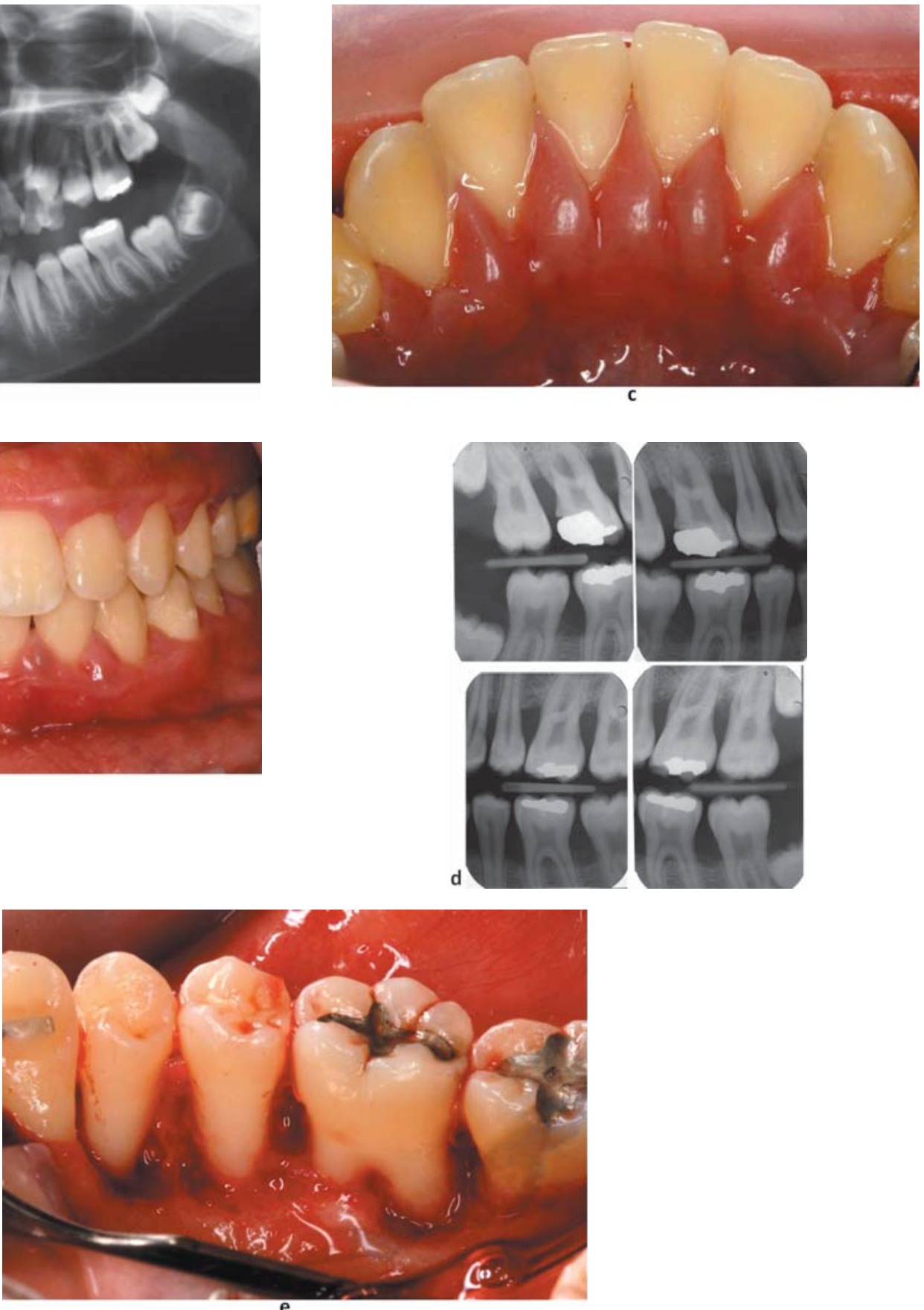

e

Figure 1- a) A panoramic view of an 11-year-old boy performed prior to orthodontic treatment; b) and c) Three years later, following orthodontic treatment, clinical presentation revealed intense gingival redness and swelling; d) Bitewing radiographs reveal marked bone loss. This rapid bone loss is pathognomonic for aggressive periodontal disease; e) Intraoperative view of one of the sextants, demonstrating the accentuated bone loss 
and lingual arch appliances. Moreover, most of the regular orthodontic treatment plans include a fixed orthodontic appliance attached to maxillary and mandibular dental arch. In this appliance molar bands or tube are often attached to first molars.

This way, it is of outmost importance to diagnose this disease as early as possible to prevent nonreversible damage to the attachment apparatus. Furthermore, the intimate contact of the orthodontic appliance including molar bands as well as retainers with the gingival tissue has a detrimental effect on oral hygiene, and bacterial plaque $8,10-12,14$ thus contributing to greater gingival inflammation in these patients (Figure 1).

Furthermore, as aggressive periodontitis usually develops during adolescence and young patients are commonly undergoing orthodontic treatment during this age with frequent visits to the orthodontic clinic, it would only make sense to call upon the orthodontist to screen for early signs of aggressive periodontitis and initiate interceptive measures. Therefore, the aim of the present paper is to draw guidelines for periodontal evaluation and monitoring prior to and during active orthodontic treatment.

\section{GUIDELINES}

In order to intercept patients with aggressive periodontitis in the orthodontic clinic as early as possible, it is recommended that periodontal examination be performed prior, during and following orthodontic treatment (Figure 2). In addition, this protocol suggests different modes of action for different periodontal conditions during the orthodontic treatment (Figure 3).

\section{BEFORE ORTHODONTIC TREATMENT}

Prior to treatment, the orthodontist must make sure the patient is capable of performing adequate oral hygiene measurements. In patients who do not comply with the required oral hygiene, active orthodontic treatment should be postponed until satisfactory plaque control is achieved. The patient should be informed that non-adherence to the oral hygiene protocol will result in discontinuation of the orthodontic treatment.

As a routine, prior to commencement of the orthodontic treatment, clinical and radiographic periodontal assessment should be performed. Clinical examination should include full-mouth probing using a standard periodontal probe,

\begin{tabular}{|c|c|c|c|}
\hline & $\begin{array}{c}\text { Before orthodontic } \\
\text { treatment }\end{array}$ & $\begin{array}{c}\text { During orthodontic } \\
\text { treatment }\end{array}$ & $\begin{array}{c}\text { Following orthodontic } \\
\text { treatment completion }\end{array}$ \\
\hline Plaque control & + & + & + \\
\hline Periodontal probing & + & + & + \\
Every 6 months & + \\
Once a year
\end{tabular}

Figure 2- Guidelines for periodontal follow-up during orthodontic care in adolescents and young adults

\begin{tabular}{|c|c|}
\hline Periodontal findings & Action required \\
\hline PD>5 mm with no BL & $\begin{array}{c}\text { Oral hygiene reinforcement; Shorten interval between } \\
\text { maintenance appointments to 4-6 weeks }\end{array}$ \\
\hline PD $>5 \mathrm{~mm}$ with $\mathrm{BL}$ & $\begin{array}{c}\text { Pause active orthodontic treatment; Refer to a } \\
\text { periodontist; Only after resolution of the periodontal } \\
\text { disease, could the orthodontic treatment continue with } \\
\text { special care and follow-up }\end{array}$ \\
\hline Gingival recession>2 $\mathrm{mm}$ & $\begin{array}{c}\text { Avoid facial tooth movements; Consider shortening } \\
\text { treatment time }\end{array}$ \\
\hline Root resorption>3 $\mathrm{mm}$ apparent in radiographs & $\begin{array}{c}\text { Consider applying lighter forces; Radiographic follow-up } \\
\text { every } 6 \text { months }\end{array}$ \\
\hline
\end{tabular}

Figure 3-Actions required following different periodontal findings. PD denotes pocket depth; BL denotes radiographic bone loss 
tooth mobility assessment and gingival recession. Radiographic evaluation should include vertical bitewing radiographs for the molar and premolar area combined with parallel periapical radiographs of the incisors (maxillary and mandibular). In subjects where pathologic periodontal pockets are observed or bone loss is detected in the radiographs, the patient should be referred for a periodontal consultation and interceptive/corrective therapy.

\section{DURING ORTHODONTIC TREATMENT}

Oral hygiene instruction and motivation should be performed after placement of the orthodontic appliances. During every visit, it is important to make sure that adequate plaque control is performed by the patient. The orthodontic appliances usually have a negative effect on oral hygiene performance thus, careful assessment and adjustment of the oral hygiene tools are necessary. Non-adherence to the oral hygiene protocols requires at least a temporary halt of the orthodontic treatment until significant improvement is evident. Periodontal recall appointments once every 3 months are advised for the period of active orthodontic treatment. This should be performed in a separate dedicated visit.

During orthodontic treatment, clinical periodontal evaluation (including periodontal probing) should be performed every 6 months. A finding of pathological pocket will require further radiographic evaluation and referral to a periodontist. Removal of the orthodontic appliance should be considered to improve periodontal outcome. After the resolution of the periodontal disease the orthodontic treatment may resume. Nonetheless, in the cases where periodontal flare-up had occurred, special care and follow-up is warranted in order to avoid recurrent periodontal disease. Radiographic examination should be performed at least once a year during the orthodontic treatment. It should include vertical bitewing radiographs for the molar and premolar area combined with parallel periapical radiographs of the incisors (maxillary and mandibular). Radiographic evaluation could also serve for caries detection and root resorption evaluation during treatment.

\section{F O L L O W I N G ORTHODONTIC TREATMENT COMPLETION}

The scheduled follow-up appointments after the completion of the orthodontic treatment might also serve as an opportunity for early detection of aggressive periodontitis in adolescence and young adults. Again, it is advised to perform quick clinical periodontal evaluation as a part of the follow-up and to combine radiographic examination once a year (vertical bitewing radiographs for the molar and premolar area combined with parallel periapical radiographs of the incisors). It should be kept in mind that orthodontic retainers, both removable and fixed, are potentially plaque retentive devices and as such have potential risk for periodontal breakdown ${ }^{8,15}$.

\section{SUMMARY AND CONCLUSIONS}

Early diagnosis and treatment are essential for successful long-term prognosis in patients with aggressive periodontitis. The role of the orthodontist in diagnosis and referral to periodontal treatment is crucial since most orthodontic patients are adolescents in which aggressive periodontitis mostly affects.

Many patients undergoing orthodontic treatment believe that their regular and frequent visits to the orthodontic office are sufficient to monitor their dental and periodontal needs. Orthodontists should be aware of this notion, which makes the fulfillment to the above guidelines of even greater importance.

Strict adherence of these guidelines as a routine protocol for periodontal examination prior, during and following orthodontic treatment may dramatically decrease the severity and improve the prognosis of aggressive periodontitis in orthodontic based clinic.

Finally, this protocol might aid the orthodontist avoiding ethical and even legal issues regarding periodontal disease detection and treatment in their patients.

\section{ACKNOWLEDGMENT}

No funding was received for this study, and the authors report no conflicts of interest.

\section{REFERENCES}

1- Albandar JM, Brown LJ, Löe H. Clinical features of early-onset periodontitis. J Am Dent Assoc. 1997;128:1393-9.

2- Albandar JM, Muranga MB, Rams TE. Prevalence of aggressive periodontitis in school attendees in Uganda. J Clin Periodontol. 2002;29:823-31.

3- Armitage GC. Development of a classification system for periodontal diseases and conditions. Ann Periodontol. 1999;4:1-6. 4- Arowojolu MO, Nwokorie CU. Juvenile periodontitis in Ibadan, Nigeria. East Afr Med J. 1997;74:372-5.

5- Harley AF, Floyd PD. Prevalence of juvenile periodontitis in schoolchildren in Lagos, Nigeria. Community Dent Oral Epidemiol. 1988; 16:299-301.

6- Kronauer E, Borsa G, Lang NP. Prevalence of incipient juvenile periodontitis at age 16 years in Switzerland. J Clin Periodontol. 1986;13:103-8.

7- Levin L, Baev V, Lev R, Stabholz A, Ashkenazi M. Aggressive periodontitis among young Israeli army personnel. J Periodontol. 2006;77:1392-6. 
8- Levin L, Samorodnitzky-Naveh GR, Machtei EE. The association of orthodontic treatment and fixed retainers with gingival health. J Periodontol. 2008;79:2087-92.

9- Löe H, Brown LJ. Early onset periodontitis in the United States of America. J Periodontol. 1991;62:608-16.

10- Lundström F, Krasse B. Streptococcus mutans and lactobacilli frequency in orthodontic patients: the effect of chlorhexidine treatment. Eur J Orthod. 1987;9:109-16.

11- Olympio KP, Bardal P, Bastos JRM, Buzalaf MAR. Effectiveness of a chlorhexidine dentifrice in orthodontic patients: a randomizedcontrolled trial. J Clin Periodontol. 2006;33:421-6.
12- Pender N. Aspects of oral health in orthodontic patients. $\mathrm{Br}$ J Orthod. 1986;13:95-103.

13- Saxby M. Prevalence of juvenile periodontitis in a British school population. Community Dent Oral Epidemiol. 1984;12:185-7.

14- Slutzkey S, Levin L. Gingival recession in young adults: occurrence, severity, and relationship to past orthodontic treatment and oral piercing. Am J Orthod Dentofacial Orthop. 2008; 13:652-6.

15- Tonetti MS, Mombelli A. Aggressive periodontitis. In: Lindhe J, Lang NP, Karring T., editors. Clinical periodontology and implant Dentistry. $5^{\text {th }}$ edition. Oxford: Blackwell; 2008. p. 428-57. 